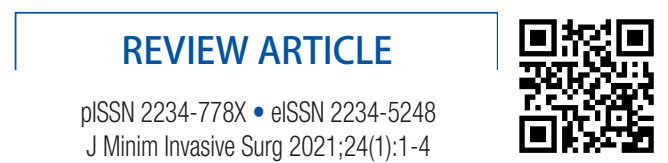

J Minim Invasive Surg 2021;24(1):1-4

\title{
Does surgical smoke matter?
}

\author{
Suk-Hwan Lee \\ Department of Surgery, Kyung Hee University Hospital at Gangdong, School of Medicine, Kyung Hee University, Seoul, Korea
}

\begin{abstract}
The coronavirus disease 2019 (COVID-19) pandemic has dramatically changed our daily lives and medical practices since it was first reported in December 2019 in Wuhan, China. Unlike the usual transmission patterns of other viral diseases, the asymptomatic or presymptomatic transmission of COVID-19 has caused difficulties in disease control around the globe. Surgical smoke or fumes may carry viruses. However, there is no evidence that the severe acute respiratory syndrome coronavirus 2, which causes COVID-19, is transmitted via surgical smoke or fumes. Laparoscopic surgeries should be continued to ensure optimal patient care. This review article aimed to investigate the current evidence relating to COVID-19 in surgical care and to discuss future remedies as the COVID-19 pandemic continues.
\end{abstract}

Keywords: COVID-19, Smoke, Laparoscopy
Received February 19, 2021

Revised March 4, 2021

Accepted March 4, 2021

Corresponding author

Suk-Hwan Lee

Department of Surgery, Kyung Hee

University Hospital at Gangdong, 892

Dongnam-ro, Gangdong-gu, Seoul

05278, Korea

Tel: $+82-2-440-6134$

Fax: +82-2-440- 6073

E-mail: leeshdr@gmail.com

ORCID:

https://orcid.org/0000-0001-6470-8620

Copyright @ 2021 The Journal of Minimally Invasive Surgery. All rights reserved.

This is an Open Access article distributed under the terms of the Creative Commons Attribution Non-Commercial License (http:// creativecommons.org/licenses/by-nc/4.0/) which permits unrestricted non-commercial use, distribution, and reproduction in any medium, provided the original work is properly cited.

\section{INTRODUCTION}

At the time of this writing, the coronavirus disease 2019 (COVID-19) pandemic has been waxing and waning throughout the country. More than 100 million people have so far been affected, and 2.3 million people have died of this disease [1,2]. The first COVID-19 case in Korea was confirmed in January 2020. Since then, there have been at least three outbreaks of COVID-19 in Korea. Despite the strict social distancing policies of the government, the daily number of affected individuals has ranged from 300 to 500 . The Korean government implemented the Korean model for COVID-19 control, which included rapid testing, rigorous contact tracing, and treating infected patients at the earliest possible stage (3Ts) [3].

At least three variants of the severe acute respiratory syndrome coronavirus 2 (SARS-CoV-2), known to cause COVID-19, have been found worldwide [4]. Some Western countries, such as the
United States and the United Kingdom, have started vaccinating their citizens.

As the COVID-19 pandemic is an extraordinary situation for all healthcare professionals and policymakers worldwide, early guidelines were not always based on evidence, as evidence was not yet available. To date, it has been more than one year since the start of the pandemic, and there is still no obvious treatment. Therefore, the protection of medical resources necessary to fight against this pandemic condition is of utmost importance. COVID-19 has impacted daily medical practices enormously, including preventive measures for the contagious disease and continuing other medical services in addition to managing COVID-19. Many strategies, such as prioritizing procedures, perioperative protocols for both infected and uninfected patients, intensive care unit (ICU) management, and prevention of viral transmission in the outpatient clinics and among admitted patients, have been implemented to keep medical services sustainable during 
the pandemic period [5]. Among the many strategies against COVID-19, this review aimed to focus on the risk of viral transmission during laparoscopic surgeries.

\section{SEVERE ACUTE RESPIRATORY SYNDROME CORONAVIRUS-2}

SARS-CoV-2 is an RNA virus, varying in size from 0.06 to 0.14 $\mu \mathrm{m}$, which causes COVID-19. Genetically, SARS-CoV-2 is highly related to SARS-CoV-1, which is responsible for the severe acute respiratory syndrome in 2003. Both viruses share the same mode of transmission via respiratory droplets [6,7]. The symptoms of COVID-19 also resemble lower respiratory infection symptoms, including fever, cough, and shortness of breath, with a median incubation period of 5 to 14 days. The loss of olfactory function was later added as a pathognomonic symptom of COVID-19.

One of the critical differences in transmission between SARSCoV-2 and SARS-CoV-1 is the high level of SARS-CoV-2 shedding in the upper respiratory tract, even among presymptomatic or asymptomatic patients. More than $50 \%$ of patients with SARSCoV-2 do not develop symptoms at the time of their screening [6]. Asymptomatic transmission of SARS-CoV-2 makes prevention and public health control of the disease worldwide more challenging.

Based on these characteristics of COVID-19, the Korean government and Korea Disease Control and Prevention Agency developed the COVID-19 prevention guidelines (Table 1).

SARS-CoV-2 is also isolated from the saliva, blood, bile, and feces. This fact is important not only for anesthetists during intubation and extubation but also for gastroenterologists and gastrointestinal and hepatobiliary surgeons. Potential transmission during standard hospital practices, such as gastrointestinal and upper airway endoscopy, is concerning and raises the fear of infection among medical staff.

Perioperative SARS-CoV-2 infection is fatal. Based on the results of an international cohort study, perioperative SARS-CoV-2 infection accounted for $51.2 \%$ of the pulmonary complication rate and $23.8 \%$ of the 30 -day mortality rate [8].

\section{DOES SMOKE MATTER IN THE TRANSMISSION OF SARS-COV-2 DURING THE SURGERY?}

The risks of laparoscopic smoke or fumes have been recognized for a long time. Owing to the hazardous chemicals, various filter systems have been introduced to minimize the risks. With the advent of the COVID-19 pandemic, the risk of aerosolization of blood-borne viruses during laparoscopic procedures has been reemphasized.

Theoretically, the high-efficiency particulate air filter is effective for filtering particles larger than $0.3 \mu \mathrm{m}$ (up to $99.97 \%$ efficacy), and the ultra-low particulate air (ULPA) filters $99.9995 \%$ of particles down to $0.1 \mu \mathrm{m}$ [9]. However, the size of SARS-CoV-2 is smaller than the limits of the ULPA filter. As such, it is impossible to remove the viruses using commercially available filters. Furthermore, commercially available ULPA filters are not currently available in South Korea.

During the early period of the COVID-19 pandemic, various measures to manage smoke during laparoscopic surgery were reported [10]. However, these measures were not practical in most hospital environments. Moreover, there is no evidence that SARS-CoV-2 is transmissible through surgical smoke.

Viruses have been isolated from surgical fumes or smoke. Previous studies have reported the presence of different viruses in the surgical smoke, including human papillomavirus (HPV), human immunodeficiency virus (HIV), and hepatitis B virus (HBV) [11-14]. Although HIV and HBV have been isolated from smoke

Table 1. Coronavirus disease 2019 (COVID-19) prevention guidelines issued by Korea Disease Control and Prevention Agency to the general public

\begin{tabular}{cl}
\hline No. & \multicolumn{1}{c}{ Guideline } \\
\hline 1 & Maintain two arms' length (2 m recommended, 1 m minimum) from other people \\
\hline 2 & Wear a mask if two arms' length cannot be maintained \\
3 & Refrain from visiting places that lack proper ventilation while many people are in proximity \\
\hline 4 & Wash your hands thoroughly with soap and running water for at least 30 seconds \\
\hline 5 & Don't touch your eyes, nose, and mouth with unwashed hands \\
\hline 6 & Cover your mouth with your sleeve or a tissue when coughing or sneezing \\
\hline 8 & Ventilate periodically throughout the day; clean and disinfect frequently touched surfaces \\
\hline 9 & Cheid contact with people who display symptoms of CoviD-19, such as fever, cough, and difficulty breathing \\
\hline 10 & Refrain from unnecessary travel \\
\hline
\end{tabular}

Resources: http://ncov.mohw.go.kr/en/. 
or laser fumes, viral disease transmission has not been reported. However, HPV transmission during anogenital surgery is widely reported in the literature. Rioux et al. [14] reported two cases of HPV 16-positive oropharyngeal squamous cell carcinoma in gynecologic surgeons who performed loop electrosurgical excision procedures. These cases occurred in healthy healthcare professionals with no risk factors for the disease, such as smoking. The only exception was long-term occupational exposure to laser plumes.

The initial guidelines from expert societies, such as the Society of American Gastroenterology and Endoscopic Surgeons and the European Association of Endoscopic Surgery, banned laparoscopic surgery during COVID-19, due to concerns related to the transmission of COVID-19 to medical staff [15]. However, the recommendation has been updated since then, as follows; "Although previous research has shown that laparoscopy can lead to aerosolization of blood-borne viruses, there is no evidence to indicate that this effect is seen with COVID-19, nor would it be isolated to minimally invasive surgery (MIS) procedures."

Considering the benefits and risks of MIS procedures to patients and medical costs, the same should be continued unless there is solid evidence of COVID-19 transmission during MIS procedures.

Keeping medical staff protected against COVID-19 is of utmost importance for securing medical services during the COVID-19 pandemic. Care should be taken to avoid transmission of COVID-19 during the perioperative period. In Korea, most hospitals require preoperative screening for COVID-19 to keep the operating theaters and staff safe from COVID-19. These strategies have recently been expanded to all admitted patients, nursing home patients, and staff.

Since the risk of viral transmission during a particular surgical procedure has remained undefined, ensuring the proper use of personal protective equipment (PPE) among surgical staff is more important and practical [5].

\section{IMPACT OF COVID-19 ON DAILY SURGICAL PRACTICES}

The integrity of medical services during the COVID-19 pandemic changed dramatically worldwide. The mortality rates among older and immunocompromised patients increased markedly during the initial stage of COVID-19. The demand for ICUs and ventilator support exceeded the readiness of the medical system in many countries.

From the experience of Northern Italy during the initial COVID-19 pandemic, the impact of COVID-19 in daily surgical practice has been widespread. Changes have ranged from the cancellation of scheduled surgeries, postponement of unnecessary surgeries to ensure available ICU care for fatal COVID-19 patients, procedural prioritization, utilization of surgeons for the COVID-19 screening process, prevention of viral transmission in the operating theaters, and stringent strategies for COVID19-dedicated operating theaters [5]. With increasing COVID-19 experience and the flattening of the COVID-19 curves over more than a year, process of medical service is getting tougher than before for all medical services including non-COVID-19 illness.

Kyung Hee University Hospital at Gangdong (KHUG), a teaching hospital in the southeast of Seoul with 800 beds, has implemented routine preoperative screening for all patients since March 2020. Since the Korean government has assigned a designated hospital for COVID-19 patients, KHUG is not responsible for the management of COVID-19 patients. However, routine preoperative screening for all operating patients still causes delays operationally, even for emergency surgeries. This policy is intended to keep the operating theaters and staff safe from COVID-19, and the operational delay of emergency surgeries has been inevitable because of the waiting time for the COVID-19 screening results. As mentioned earlier in the manuscript, among the 3Ts (test, tracing, and treatment) of the Korean governmental strategies, testing is the core component of COVID-19 prevention in Korea [3]. The reverse transcription-polymerase chain reaction test results are obtained after approximately 3 hours, depending on the sampling time. In cases of appendicitis, the waiting interval, defined as the time interval from the admission to operation before the routine COVID-19 test, was on average 11.8 hours (11.8 \pm 7.8 hours; range, 2.7-38.5 hours) compared to that of 16.8 hours (16.8 \pm 8.5 hours; range, $2.8-54.5$ hours) $(p<0.001)$ after the routine screening test (unpublished data). At least 5 hours have been added by waiting for the screening test results from routine preoperative screening for COVID-19. Fortunately, the severity of appendicitis has not been compromised during the waiting period because intravenous antibiotics are started as soon as the patient is admitted to the hospital.

A plan for prioritizing surgery should be prepared depending on the COVID-19 pandemic situation. If medical resources are sufficient to accommodate COVID-19 patients, routine surgical planning is possible. In the event of a COVID-19 outbreak, each hospital should prepare its own prioritization policies for all surgical procedures. If the prevalence of COVID-19 is tolerable by the community and hospital resources can cope with the demand for ward and ICU care, most elective surgeries should be allowed to continue as usual. During the peak outbreak period, surgeries should be reserved for inevitable life-threatening conditions, such as perforated hollow viscus or active bleeding cases, or septic patients who need operative interventions.

\section{CONCLUSIONS}

COVID-19 has caused many unexpected situations in medical 
care and social behaviors. The initial fear of the deadly COVID-19 pandemic led to some unnecessary recommendations that were not based on evidence. Healthcare professionals should be aware of the COVID-19 condition and aim to maintain the integrity of medical services. There is no evidence that surgical smoke transmits SARS-CoV-2 during or after laparoscopic surgery. However, caution should be taken to prevent potential COVID-19 transmission by using appropriate PPE and ensuring a plan for the prioritization of surgeries.

\section{NOTES}

\section{Conflicts of interest}

The author has no conflicts of interest to declare.

\section{REFERENCES}

1. Ministry of Health and Welfare (MOHW). COVID-19 statistics, Republic of Korea [Internet]. Sejong (Korea): MOHW; 2021 [cited 2021 Feb 3]. Available from: http://ncov.mohw.go.kr/en/

2. World Health Organization (WHO). Coronavirus disease (COVID-19) pandemic [Internet]. Geneva: WHO; 2020 [cited 2021. Feb 3]. Available from: https://www.who.int/emergencies/diseases/novelcoronavirus-2019.

3. Park Y, Huh IS, Lee J, et al. Application of testing-tracing-treatment strategy in response to the COVID-19 outbreak in Seoul, Korea. J Korean Med Sci 2020;35:e396.

4. Mahase E. Covid-19: what have we learnt about the new variant in the UK? BMJ 2020;371:m4944.

5. Al-Jabir A, Kerwan A, Nicola M, et al. Impact of the coronavirus (COVID-19) pandemic on surgical practice: part 1. Int J Surg 2020;79:
168-179.

6. Arons MM, Hatfield KM, Reddy SC, et al. Presymptomatic SARS$\mathrm{CoV}-2$ infections and transmission in a skilled nursing facility. N Engl J Med 2020;382:2081-2090.

7. Gandhi M, Yokoe DS, Havlir DV. Asymptomatic transmission, the Achilles' heel of current strategies to control covid-19. N Engl J Med 2020;382:2158-2160.

8. COVIDSurg Collaborative. Mortality and pulmonary complications in patients undergoing surgery with perioperative SARS-CoV-2 infection: an international cohort study. Lancet 2020;396:27-38.

9. Mowbray NG, Ansell J, Horwood J, et al. Safe management of surgical smoke in the age of COVID-19. Br J Surg 2020;107:1406-1413.

10. MIS Filtration Group. How to manage smoke evacuation and filter pneumoperitoneum during laparoscopy to minimize potential viral spread: different methods from SoMe: a video vignette. Colorectal Dis 2020;22:644-645.

11. Baggish MS, Poiesz BJ, Joret D, Williamson P, Refai A. Presence of human immunodeficiency virus DNA in laser smoke. Lasers Surg Med 1991;11:197-203.

12. Kwak HD, Kim SH, Seo YS, Song KJ. Detecting hepatitis B virus in surgical smoke emitted during laparoscopic surgery. Occup Environ Med 2016;73:857-863.

13. Liu Y, Song Y, Hu X, Yan L, Zhu X. Awareness of surgical smoke hazards and enhancement of surgical smoke prevention among the gynecologists. J Cancer 2019;10:2788-2799.

14. Rioux M, Garland A, Webster D, Reardon E. HPV positive tonsillar cancer in two laser surgeons: case reports. J Otolaryngol Head Neck Surg 2013;42:54.

15. Francis N, Dort J, Cho E, et al. SAGES and EAES recommendations for minimally invasive surgery during COVID-19 pandemic. Surg Endosc 2020;34:2327-2331. 